\title{
$\alpha$-Helical nascent polypeptide chains visualized within distinct regions of the ribosomal exit tunnel
}

\author{
Shashi Bhushan ${ }^{1,5}$, Marco Gartmann ${ }^{1,5}$, Mario Halic ${ }^{1,4}$, Jean-Paul Armache ${ }^{1}$, Alexander Jarasch ${ }^{1}$, \\ Thorsten Mielke ${ }^{2,3}$, Otto Berninghausen ${ }^{1}$, Daniel N Wilson ${ }^{1} \&$ Roland Beckmann $^{1}$
}

As translation proceeds, the nascent polypeptide chain passes through a tunnel in the large ribosomal subunit. Although this ribosomal exit tunnel was once thought only to be a passive conduit for the growing nascent chain, accumulating evidence suggests that it may in fact play a more active role in regulating translation and initial protein folding events. Here we have determined single-particle cryo-electron microscopy reconstructions of eukaryotic $80 \mathrm{~S}$ ribosomes containing nascent chains with high $\alpha$-helical propensity located within the exit tunnel. The maps enable direct visualization of density for helices as well as allowing the sites of interaction with the tunnel wall components to be elucidated. In particular regions of the tunnel, the nascent chain adopts distinct conformations and establishes specific contacts with tunnel components, both ribosomal RNA and proteins, that have been previously implicated in nascent chain-ribosome interaction.

The ribosomal exit tunnel is a universal feature of ribosomes ${ }^{1-4}$. The tunnel is $80-100 \AA$ long and 10-20 $\AA$ wide and is composed predominantly of ribosomal RNA (rRNA), consistent with an overall electronegative potential ${ }^{5,6}$. Along with rRNA, the extensions of the ribosomal proteins L4 and L17 (L22 in bacteria) contribute to formation of the tunnel wall and form a so-called 'constriction' where the tunnel narrows ${ }^{2}$. The ribosomal protein L39 is placed near the tunnel exit in eukaryotic and archaeal ribosomes, whereas in bacteria the extension of L23 (L25 in eukaryotes) occupies a similar position.

Despite its universality, a functional role for the ribosomal tunnel is only beginning to emerge. For many years, the ribosomal tunnel was believed to be only a passive conduit for the nascent polypeptide chain. However, accumulating evidence indicates that, at least for some nascent chains, the tunnel has a more active role. In particular, a number of leader peptides induce translational stalling in response to the presence or absence of an effector molecule and, in doing so, regulate translation of a downstream gene ${ }^{7}$. Three well-characterized examples include the bacterial SecM, TnaC and ErmC leader peptides, for which mutations in the leader peptide sequence, or in the ribosomal tunnel components themselves, can relieve the translational arrest ${ }^{8-10}$. Collectively, these data imply a direct interaction between specific residues of the leader peptide with distinct locations in the ribosomal tunnel.

The dimensions of the tunnel preclude the folding of domains as large as an IgG domain $(\sim 17 \mathrm{kDa})^{11}$, whereas $\alpha$-helix formation is more feasible ${ }^{11,12}$. Indeed, fluorescence resonance energy transfer (FRET) studies have indicated that a transmembrane sequence is compacted as it travels through the ribosome, consistent with $\alpha$-helix formation in the tunnel ${ }^{13}$. Interestingly, in the same study, the compaction of the transmembrane nascent chain was lost upon exciting the tunnel, suggesting that the tunnel plays a pivotal role in stabilizing the proposed helical conformation. Independent biochemical analyses also provide support for the potential of the nascent chain to adopt compacted or helical conformations in the tunnel and have even identified specific regions of the tunnel that promote compaction ${ }^{14-17}$.

To gain direct structural insight as to whether it is possible for an $\alpha$-helix to form within the ribosomal exit tunnel, we used cryoelectron microscopy and single-particle reconstruction to determine two structures of ribosome complexes bearing nascent chains with regions of high $\alpha$-helical propensity. Our results enable us to directly visualize density consistent with $\alpha$-helix formation in the tunnel as well as the sites of interaction between the nascent chain and tunnel wall components. $\alpha$-helix formation within distinct regions of the ribosomal exit tunnel may have implications for membrane insertion and co-translational protein folding.

\section{RESULTS}

Generation of 80S-helix ribosome-nascent chain complexes As in previous studies ${ }^{18,19}$, we have used a Triticum aestivum (wheat germ) in vitro translation system to prepare $80 \mathrm{~S}$ ribosome-nascent chain complexes (RNCs) with dipeptidylaminopeptidase B (DPAP-B) nascent chain in the tunnel. We truncated the DNA templates to remove the stop codon, thus trapping the translating ribosomes at the last codon. In this study, we generated constructs by replacing two different parts of the DPAP-B sequence with a short peptide that has a strong propensity to form a hydrophilic $\alpha$-helix in solution ${ }^{20,21}$

${ }^{1}$ Gene Center and Center for Integrated Protein Science Munich, Department of Biochemistry, University of Munich, Munich, Germany. ${ }^{2}$ UltraStrukturNetzwerk, Max Planck Institute for Molecular Genetics, Berlin, Germany. ${ }^{3}$ Institut für Medizinische Physik und Biophysik, Charité, Berlin, Germany. ${ }^{4}$ Present address: Harvard Medical School, Department of Cell Biology, Boston, Massachusetts, USA. ${ }^{5}$ These authors contributed equally to this work. Correspondence should be addressed to R.B. (beckmann@Imb.uni-muenchen.de). 
Figure 1 Cryo-electron microscopy reconstructions of RNCs. (a-f) Cryo-electron microscopy reconstructions of the 80S-helix 1 RNC (a,b), 80S-helix2 RNC (c,d) and 80S$\operatorname{DPAP} \operatorname{RNC}(\mathbf{e}, \mathbf{f})$. For each reconstruction, the top left inset shows the overall orientation of the small (40S, yellow) and large (60S, gray) subunits, with respective cross-sections $(\mathbf{a}, \mathbf{c}, \mathbf{e})$ and tunnel views $(\mathbf{b}, \mathbf{d}, \mathbf{f})$, below. Right-hand panels show zoom of the respective crosssections and tunnel views of each complex. Densities attributed to the P-tRNA and nascent chain in the 80S-helix1 RNC, 80S-helix2 RNC and 80S-DPAP RNC are colored gold, blue and green, respectively.

(Supplementary Fig. 1). The peptide contains five Glu-Ala-Ala-Ala-Lys (EAAAK) repeats and forms a standard $[i+4 \rightarrow i]$ $\alpha$-helix, in which every backbone $\mathrm{N}-\mathrm{H}$ group donates a hydrogen bond to the backbone $\mathrm{C}=\mathrm{O}$ group of the amino acid four residues earlier (Supplementary Fig. 1). In addition, each repeat of the helix is stabilized by a Glu ${ }^{-}$-Lys $^{+}$salt bridge, leading to $>80 \%$ helicity in aqueous solvent as determined by circular dichroism studies ${ }^{20,21}$. When translation reaches the $3^{\prime}$ end of the truncated mRNA, 115 residues have been translated and asparagine-tRNA is located at the P site of the ribosome. In the helix 1 construct, the helix-forming sequence is positioned at residues 72-96, whereas in helix 2 it is located at residues $83-108,-19$ and -7 from the asparagine of the peptidyl-tRNA (P-tRNA), respectively (Supplementary Fig. 1). Because the ribosomal tunnel is known to protect $30-40$ residues $^{12}$, both helix-forming sequences should still be enclosed within the exit tunnel.

\section{Visualization of 80S-helix RNCs}

We then applied cryo-electron microscopy and single-particle analysis to reconstruct the two 80S RNCs each with the helix-forming

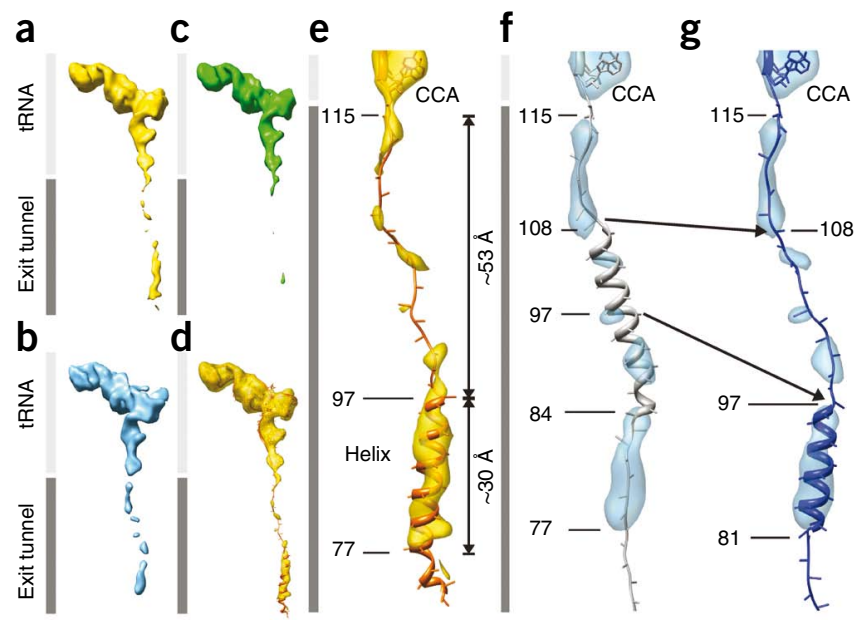

Figure 2 Comparison of tRNA-nascent chains from RNCs. Isolated density for the P-site tRNA and nascent chain from the 80S-helix1 RNC (a, gold), 80S-helix2 RNC (b, blue) and 80S-DPAP RNC (c, green). (d) Transparent density of a with fitted ribbon model for tRNA and nascent chain. (e) Zoom of $\mathbf{d}$ with residue numbering, as well as distances between $\mathrm{C} \alpha$ of residues 97-115 and 77-97. CCA indicates the position of the terminal CCA end of the P-tRNA. $(\mathbf{f}, \mathbf{g})$ Zoom of $\mathbf{b}$ with alternative models for the helix2 nascent chain. Arrows indicate corresponding region (residues 97-108) that is modeled as helical (f) or extended (g). sequences in the tunnel (80S-helix1 RNC and 80S-helix2 RNC; Fig. 1a-d and Supplementary Fig. 1), as well as the control $80 \mathrm{~S}$ RNC bearing the DPAP-B sequence (80S-DPAP RNC; Fig. 1e,f), at resolutions of 7.1-7.3 $\AA$ (0.5 criterion; Supplementary Fig. 2). In all reconstructions, we observed strong density for a single P-tRNA within the intersubunit space (Fig. 1). Most striking, however, was the presence of additional density within the exit tunnel of the $80 \mathrm{~S}$ helix RNCs that can be attributed to the nascent chain (Fig. 1a-d), whereas in comparison, the tunnel of the control 80S-DPAP RNC contains little additional density for the corresponding DPAP-B nascent chain (Fig. 1e,f). In the tunnel of the 80S-helix RNCs, the strongest region of density is observed for the $\mathrm{N}$-terminal region of the nascent chain near the tunnel exit (Fig. 1a-d).

We isolated the densities for the P-tRNA and associated nascent chains of the 80S-helix RNCs and 80S-DPAP RNC (Fig. 2a-c) and then fit a molecular model for the P-tRNA to the isolated density of the 80S-helix1 RNC (Fig. 2d). Locating the terminal CCA end of the tRNA in turn identifies the $\mathrm{C}$ terminus of the nascent chain. All three reconstructions have some density corresponding to the nascent chain directly adjacent to the peptidyltransferase center (PTC) (Fig. 2). In the 80S-helix1 RNC, the strong density for the $\mathrm{N}$-terminal region of the nascent chain is consistent with an $\alpha$-helical conformation in this region and coincides perfectly with the expected location in the tunnel (Fig. 2d,e and Supplementary Fig. 3). Because the EAAAK repeats have been structurally characterized in the antifreeze proteins of cold-water fish such as the winter flounder, we were able to build a homology model for the helical part of the nascent chain based on the known crystal structure of the antifreeze protein HPLC6 isoform ${ }^{22}$ (Fig. 2d,e and Supplementary Fig. 1). The density can account for four of the five EAAAK repeats, correlating to 20 residues or six turns of the $\alpha$-helix (3.3 residues per turn) (Fig. 2e). At lower thresholds, additional density extends into the solvent from the N-terminal part of the helix, most likely indicating flexibility in this region. We do not think the $\alpha$-helix unwinds upon exiting the tunnel, as suggested by FRET studies with a transmembrane helix ${ }^{13}$, as the EAAAK repeats and antifreeze peptides have been shown by circular dichroism and NMR to be stable in solution ${ }^{20,21,23}$. With this fitting for the helix, the proximal portion of the helix (Gly97) is located $\sim 53 \AA$ from Asp1 15 at the P site, and it is thus only possible to span this distance using an extended conformation (3.0-3.4 $\AA$ per residue) for the 18 intervening residues of the nascent chain (Fig. 2e). The properties of the observed density for these regions are also consistent with an extended conformation for these residues, 
Figure 3 Helix-nascent chain interactions with tunnel components. Cross-section of the upper (left panels) and lower (right panels) tunnel regions of the 80S-helix1 RNC (a-d, gold), 80S-helix2 RNC (e,f, blue) and comparison of both $(\mathbf{g}, \mathbf{h})$. Sites of contacts between nascent chain and ribosomal tunnel components are indicated with asterisks $\left({ }^{*}\right)$. The cryo-electron microscopy map of the 80S-helix1 RNC and 80S-helix2 RNC are shown in gray, with isolated density for the nascent chain (gold and blue mesh, respectively) shown at a lower threshold. The $28 \mathrm{~S}$ rRNA nucleotides are shown as blue sticks, whereas ribosomal proteins L4 (green), L17 (blue) and L39 (magenta) are displayed as ribbons. In a and c, nucleotide A2062 ( $E$. coli numbering) is shown in two conformations, taken from PDB 1 S72 (ref. 2; blue) and PDB 1VQN (ref. 24; red).

whereas the fragmentation suggests that the nascent chain does not adopt a distinct conformation in some sections of the tunnel.

Similarly to the 80 S-helix1 RNC, in the 80 S-helix2 RNC we observed the strongest density for the nascent chain in the lower region of the exit tunnel (Fig. 2f). However, if all five repeats of the EAAAK sequence had adopted a helical conformation, we would have expected strong density to also be present in the middle of the tunnel. Therefore, it is likely that the proximal portion of the region with helical propensity (residues 97-108) is unable to adopt an $\alpha$-helical conformation but instead acquires an extended conformation. Although some compaction in the middle region of the tunnel might not be observed due to flexibility, this possibility is incompatible with the excellent agreement between the density and the model for the distal portion of the remaining helical stretch (residues 81-97) (Fig. 2g). Curiously, more density for the nascent chain is observed in the upper region (residues 108-115) of the 80S-helix2 RNC compared to the 80S-helix1 RNC, suggesting an influence of the helix 2 sequence on the conformation of the adjacent region of the nascent chain. Although there appears to be slightly more compaction within this region of the 80 S-helix 2 RNC, the density is not consistent with a helix (Supplementary Fig. 4).

\section{Nascent chain-ribosome interactions within the exit tunnel}

To dissect the contacts between the nascent chain and ribosomal components of the exit tunnel, we generated and then fitted a molecular model of the wheat germ ribosomal tunnel to both the 80S-helix RNC maps (Fig. 3a-h and Supplementary Fig. 5). In the upper region of the tunnel of the 80S-helix1 RNC, we observed three connections with strong density between the nascent chain and the tunnel wall (Fig. 3a). Adjacent to the PTC, the nascent chain appeared to form a contact in the vicinity of 28S rRNA nucleotide A2062 (Escherichia coli numbering used throughout). This contact did not appear to be present in the 80S-helix2 RNC (Fig. 3e). From the available ribosome crystal structures, A2062 is known to be flexible, adopting a position flat against the tunnel wall, as in the Haloarcula marismortui apo-50S structure ${ }^{2}$, or protruding into the tunnel lumen when P-site tRNA mimics are bound ${ }^{24}$. Clearly, the latter position fits better to the density of the 80S-helix1 RNC map, suggesting that the nascent chain stabilizes a distinct conformation of A2062 (Fig. 3a). In E. coli, mutations at A2062 relieve the translational arrest resulting from ErmC stalling ${ }^{10}$.

Deeper in the tunnel, the nascent chain established contact in the region of nucleotide A751 in both 80S-helix RNC maps (Fig. 3b,f). Insertions at $\mathrm{A} 751$ have been reported to eliminate tryptophan induction associated with TnaC-mediated translational stalling ${ }^{25}$. The extensions of ribosomal protein L4 formed two contacts with the 80S-helix1 RNC nascent chain, one near the constriction (Fig. 3a) and a second to the proximal end of the helix (Fig. 3b), whereas in 80S-helix2 RNC the proximal contact was absent (Fig. 3f) and the L4 contact at a

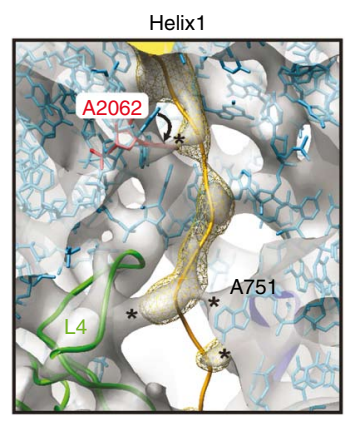

C

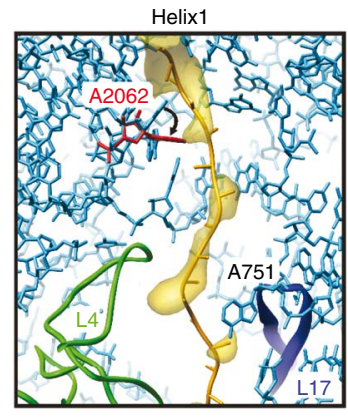

e

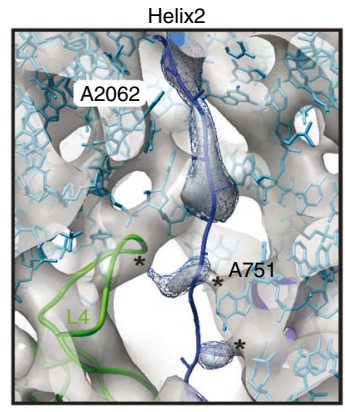

g

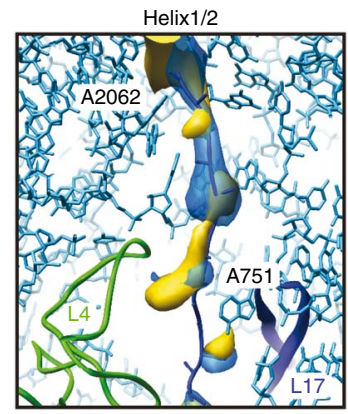

b

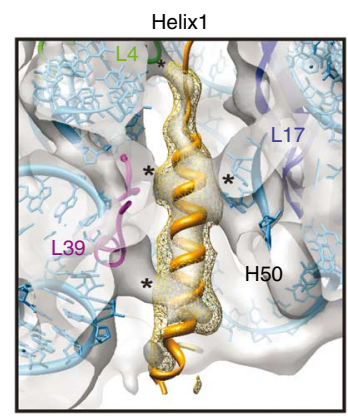

d

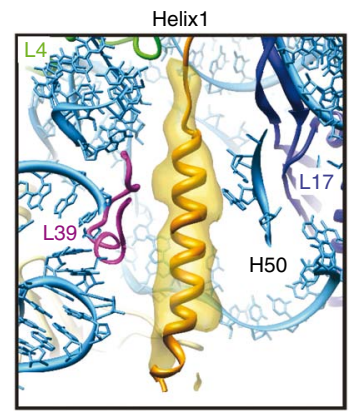

f

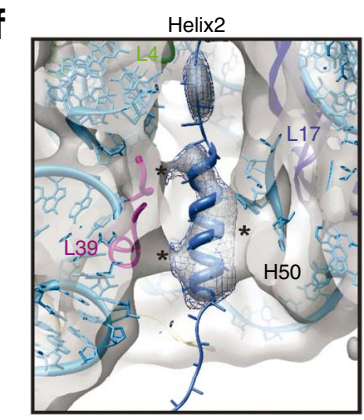

$\mathbf{h}$

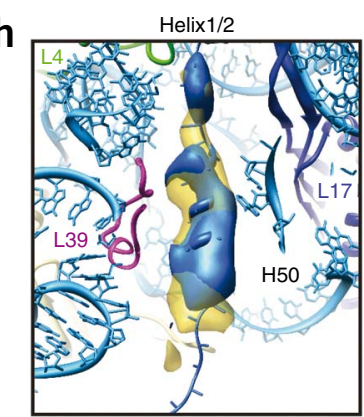

the constriction is represented in the map by substantially weaker density (Fig. 3e). We observed negligible density between these two points for both nascent chains, indicating that they did not adopt a single distinct conformation in this region (Fig. 3a,e). Despite this, we observed additional density contacting the tip of the $\beta$-hairpin of L17 (Fig. 3a,e). A compacted transmembrane containing nascent chain photocross-links to L17 (ref. 13), and in E. coli, mutations within this region of L22 (the homolog of L17) can relieve TnaC- and SecM-mediated stalling ${ }^{9,25}$. The fact that the contacts observed here for nonstalling sequences are similar in location to those predicted for some of the known stalling leader peptides hints that these regions of the tunnel represent functional hot spots for tunnel-nascent chain interaction.

\section{$\alpha$-Helix formation in the ribosomal exit tunnel}

In both 80 S-helix RNC maps, the density for the $\alpha$-helix is sandwiched between ribosomal protein L39 and the loop region of helix 


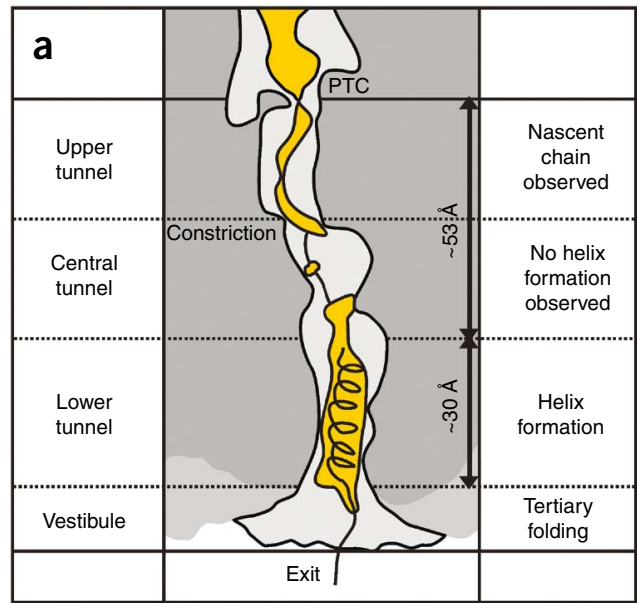

b

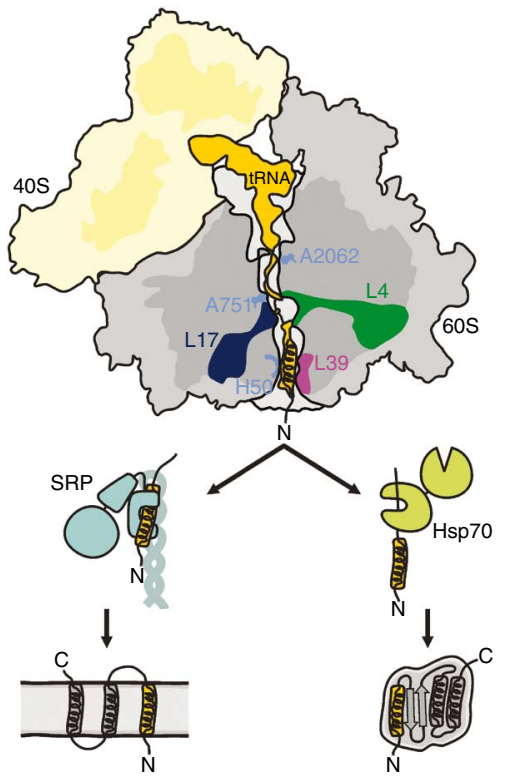

50 (H50) of the 28S rRNA (Fig. 3b,c). L39 appears to make two strong contacts with the helix, one proximal and the other more distal (N-terminal), whereas the H50 contact appears to be different between the two structures (Fig. 3b). Photo-cross-linking studies have identified sequential contacts of a compacted transmembrane containing nascent chain, first to L4, then to L17 and finally to L39 (ref. 13)-ribosomal proteins that are visualized to contact the nascent chain in both of the 80S-helix RNCs (Fig. 4a). In these studies, it was concluded that the transmembrane helix maintains a helical conformation during its passage throughout the entire ribosomal tunnel. Although we observed no helix formation in the middle of the tunnel (that is, directly following the constriction (Fig. 4a)), the observed discrepancy can be related to the use of a hydrophobic transmembrane helix ${ }^{13}$ rather than the hydrophilic helix used here. In any case, the ability to form a helix near to the tunnel exit site but not in the middle region of the tunnel is consistent with the zones of secondary-structure formation identified previously ${ }^{15}$.

\section{DISCUSSION}

The fact that $\alpha$-helix formation within the exit tunnel is indeed possible in distinct regions raises the question of what the functional significance of this finding might be. First, it is tempting to speculate
Figure 4 Implications of helix formation within the ribosomal exit tunnel. (a) Schematic representation of a cross-section of the 80S-helix1 RNC showing the regions where helix and tertiary structure formation are observed. (b) Schematic representation of a cross-section of the 80S-helix1 RNC complex, illustrating sites of contact between the nascent chain (gold) and ribosomal components; $28 \mathrm{~S}$ rRNA nucleotides A2062 and A751, ribosomal proteins L4 (green), L17 (blue) and L39 (magenta). The helix of the nascent chain forms interactions with helix 50 (H50) of the 28S rRNA and L39. Helix formation in the ribosomal tunnel may assist in membrane insertion, as shown for signalrecognition particle (SRP)-dependent pathway, and/or may promote the correct or more efficient folding of cytoplasmic proteins, with or without the aid of chaperones such as heat shock protein 70 (Hsp70).

that allowing, or even promoting ${ }^{15}, \alpha$-helix formation when $\beta$-sheet formation is not yet possible has an impact on protein folding. This would imply that folding might occur using a hierarchy of secondarystructure elements, with $\alpha$-helix formation occurring first wherever possible. Such a scenario would considerably reduce the complexity of the theoretical conformational space that must be sampled before the correct fold is adopted. Additionally, it would also change the appearance of nascent peptides as substrates for co-translationally acting chaperones (Fig. 4b). Indeed, tertiary structure formation, such as the formation of $\alpha$ - and $\beta$-hairpins, has already been observed to occur near the tunnel exit ( $>80 \AA$ from tunnel start), where the tunnel widens substantially to form a vestibule ${ }^{5,26}$ (Fig. 4a).

Second, $\alpha$-helix formation in the tunnel may be important for proteins containing $\alpha$-helical domains destined for membrane insertion ${ }^{13,27}$. Co-translational targeting by signal-recognition particle (SRP), for example, may be promoted, as (i) the presence of a signal-anchor sequence within the tunnel promotes binding of SRP to the ribosome $e^{28}$ and (ii) $\alpha$-helicity of the signal sequence is important for its recognition by $\mathrm{SRP}^{29}$ (Fig. 4b). Indeed, compaction of transmembrane domains in the ribosomal tunnel has been reported ${ }^{13,16}$, and a compacted conformation for the signalanchor sequence has been observed by cryo-electron microscopy to bind in the vestibule at the end of the ribosomal tunnel on E. coli ribosomes ${ }^{19}$.

Third, restricting $\alpha$-helix formation to only certain regions of the tunnel may reduce the modes of interaction of the nascent peptide with the tunnel wall. Compared to an extended chain, a nascent chain forming an $\alpha$-helix throughout the entire tunnel could potentially establish twice as many interactions. This might result in unfavorably stable interactions. On the other hand, helix formation in permitting regions may help to scan for distinct interaction patterns facilitating regulatory events. These hypotheses need to be examined, but the conservation of the dimensions of the ribosomal tunnel is consistent with its significance in providing nascent proteins with a very defined first environment.

\section{METHODS}

Methods and any associated references are available in the online version of the paper at http://www.nature.com/nsmb/.

Accession codes. The cryo-electron microscopic maps of the 80S-helix1 RNC, 80S-helix2 RNC and 80S-DPAP RNC have been deposited in the 3D-EM database under accession numbers EMD-1664, EMD-1665 and EMD-1666, respectively.

Note: Supplementary information is available on the Nature Structural \& Molecular Biology website. 


\section{ACKNOWLEDGMENTS}

We would like to thank B. Beatrix for help with the wheat germ translation system and J. Buerger for help with the electron microscopy. This research was supported by Federation of European Biochemical Societies and Knut och Alice Wallenbergs Stiftelse postdoctoral fellowships (to S.B.), grants from the Deutsche Forschungsgemeinschaft SFB594 and SFB646 (to R.B.), SFB740 (to T.M.) and WI3285/1-1 (to D.N.W.) and by the European Union and Senatsverwaltung für Wissenschaft, Forschung und Kultur Berlin.

\section{AUTHOR CONTRIBUTIONS}

S.B., M.G. and R.B. designed research; S.B. prepared the complexes and collected data; O.B. and T.M. helped with data collection; S.B., M.G. and M.H. processed datasets; J.-P.A., A.J. and D.N.W. prepared models; S.B., D.N.W. and R.B. analyzed results, prepared figures and wrote the paper.

\section{COMPETING INTERESTS STATEMENT}

The authors declare no competing financial interests.

Published online at http://www.nature.com/nsmb/.

Reprints and permissions information is available online at http://npg.nature.com/ reprintsandpermissions/.

1. Frank, J. et al. A model of protein synthesis based on cryo-electron microscopy of the $E$. coli ribosome. Nature 376, 441-444 (1995).

2. Ban, N., Nissen, P., Hansen, J., Moore, P.B. \& Steitz, T.A. The complete atomic structure of the large ribosomal subunit at $2.4 \AA$ resolution. Science 289, 905-920 (2000)

3. Morgan, D.G. et al. A comparison of the yeast and rabbit $80 \mathrm{~S}$ ribosome reveals the topology of the nascent chain exit tunnel, inter-subunit bridges and mammalian rRNA expansion segments. J. Mol. Biol. 301, 301-321 (2000).

4. Halic, M. et al. Signal recognition particle receptor exposes the ribosomal translocon binding site. Science 312, 745-747 (2006).

5. Lu, J. \& Deutsch, C. Electrostatics in the ribosomal tunnel modulate chain elongation rates. J. Mol. Biol. 384, 73-86 (2008).

6. Lu, J., Kobertz, W.R. \& Deutsch, C. Mapping the electrostatic potential within the ribosomal exit tunnel. J. Mol. Biol. 371, 1378-1391 (2007).

7. Tenson, T. \& Ehrenberg, M. Regulatory nascent peptides in the ribosomal tunnel. Cell 108, 591-594 (2002).

8. Gong, F. \& Yanofsky, C. Instruction of translating ribosome by nascent peptide. Science 297, 1864-1867 (2002)

9. Nakatogawa, H. \& Ito, K. The ribosomal exit tunnel functions as a discriminating gate. Cell 108, 629-636 (2002).

10. Vazquez-Laslop, N., Thum, C. \& Mankin, A.S. Molecular mechanism of drugdependent ribosome stalling. Mol. Cell 30, 190-202 (2008).
11. Voss, N.R., Gerstein, M., Steitz, T.A. \& Moore, P.B. The geometry of the ribosomal polypeptide exit tunnel. J. Mol. Biol. 360, 893-906 (2006).

12. Hardesty, B. \& Kramer, G. Folding of a nascent peptide on the ribosome. Prog. Nucleic Acid Res. Mol. Biol. 66, 41-66 (2001).

13. Woolhead, C.A., McCormick, P.J. \& Johnson, A.E. Nascent membrane and secretory proteins differ in FRET-detected folding far inside the ribosome and in their exposure to ribosomal proteins. Cell 116, 725-736 (2004).

14. Kosolapov, A., Tu, L., Wang, J. \& Deutsch, C. Structure acquisition of the T1 domain of Kv1.3 during biogenesis. Neuron 44, 295-307 (2004).

15. Lu, J. \& Deutsch, C. Folding zones inside the ribosomal exit tunnel. Nat. Struct Mol. Biol. 12, 1123-1129 (2005).

16. Lu, J. \& Deutsch, C. Secondary structure formation of a transmembrane segment in Kv channels. Biochemistry 44, 8230-8243 (2005).

17. Woolhead, C.A., Johnson, A.E. \& Bernstein, H.D. Translation arrest requires two-way communication between a nascent polypeptide and the ribosome. Mol. Cell 22, 587-598 (2006).

18. Halic, M. et al. Structure of the signal recognition particle interacting with the elongation-arrested ribosome. Nature 427, 808-814 (2004).

19. Halic, M. et al. Following the signal sequence from ribosomal tunnel exit to signa recognition particle. Nature 444, 507-511 (2006)

20. Marqusee, S. \& Baldwin, R.L. Helix stabilization by Glu $^{-}$.Lys ${ }^{+}$salt bridges in short peptides of de novo design. Proc. Natl. Acad. Sci. USA 84, 8898-8902 (1987).

21. Arai, R., Ueda, H., Kitayama, A., Kamiya, N. \& Nagamune, T. Design of the linkers which effectively separate domains of a bifunctional fusion protein. Protein Eng. 14, 529-532 (2001).

22. Sicheri, F. \& Yang, D.S. Ice-binding structure and mechanism of an antifreeze protein from winter flounder. Nature 375, 427-431 (1995).

23. Liepinsh, E. et al. Solution structure of a hydrophobic analogue of the winte flounder antifreeze protein. Eur. J. Biochem. 269, 1259-1266 (2002)

24. Schmeing, T.M., Huang, K.S., Strobel, S.A. \& Steitz, T.A. An induced-fit mechanism to promote peptide bond formation and exclude hydrolysis of peptidyl-tRNA. Nature 438, 520-524 (2005).

25. Cruz-Vera, L.R., Rajagopal, S., Squires, C. \& Yanofsky, C. Features of ribosomepeptidyl-tRNA interactions essential for tryptophan induction of tha operon expression. Mol. Cell 19, 333-343 (2005).

26. Kosolapov, A. \& Deutsch, C. Tertiary interactions within the ribosomal exit tunnel. Nat. Struct. Mol. Biol. 16, 405-411 (2009).

27. Liao, S., Lin, J., Do, H. \& Johnson, A.E. Both lumenal and cytosolic gating of the aqueous ER translocon pore are regulated from inside the ribosome during membrane protein integration. Cell 90, 31-41 (1997).

28. Berndt, U., Oellerer, S., Zhang, Y., Johnson, A.E. \& Rospert, S. A signal-anchor sequence stimulates signal recognition particle binding to ribosomes from inside the exit tunnel. Proc. Natl. Acad. Sci. USA 106, 1398-1403 (2009).

29. Mingarro, I., Nilsson, I., Whitley, P. \& von Heijne, G. Different conformations of nascent polypeptides during translocation across the ER membrane. BMC Cell Biol. 1, 3 (2000). 


\section{ONLINE METHODS}

RNC preparation. The helixl construct was chemically synthesized by Eurofins. We prepared DNA template for transcription using T7 standard and reverse (5'-ATCGTAGACAGATTTAACAAC-3') primers by PCR. We generated the helix 2 construct by PCR using a standard T7 primer with a modified reverse (5'-ATCGTACTCGAGACCACCAGCTTTAG-3') primer, with helix1 construct as the template. We then synthesized uncapped transcripts from the PCR fragments using T7 RNA polymerase. We generated 80S-helix RNCs using a self-made wheat germ in vitro translation system ${ }^{30}$ programmed with truncated mRNA encoding the helix 1 and 2 regions. We purified RNCs as described previously ${ }^{18}$. We generated and purified 80S-DPAP RNCs as described previously ${ }^{18}$.

Electron microscopy, image processing and modeling. According to standard methods ${ }^{31}$, we applied samples to carbon-coated holey grids. We recorded micrographs under low-dose conditions on a Tecnai F30 field emission gun electron microscope at $300 \mathrm{kV}$ in a defocus range of $1.0-4.5 \mu \mathrm{m}$ and scanned on a Heidelberg drum scanner, resulting in a pixel size of $1.24 \AA$ on the object scale. We analyzed the data by determination of the contrast transfer function (CTF) using CTFFIND ${ }^{32}$. We further processed the data with the SPIDER software package $^{33}$. After automated particle picking followed by visual inspection, we selected 200,000 particles for 80S-helix1 RNC, 230,000 particles for 80S-helix2 RNC and 250,000 for 80S-DPAP RNC for density reconstruction. We sorted the data into programmed (with P-site tRNA) and unprogrammed or empty ribosome (without P-site tRNA) subdatasets using reconstructions of programmed and unprogrammed ribosomes as references (for example, see Supplementary Fig. 6). Removal of empty ribosomal particles resulted in 120,000, 190,000 and 150,000 programmed particles for 80S-helix1 RNC, 80S-helix2 RNC and 80S-DPAP RNC, respectively, yielding the final CTF-corrected reconstruction at a resolution of $7.1 \AA, 7.3 \AA$ and $7.1 \AA$, respectively, based on the Fourier shell correlation with a cutoff value of 0.5 (Supplementary Fig. 2). We isolated densities (for example, see Supplementary Fig. 7) for the 40 S subunit, the 60 S subunit and the P-site tRNA by using binary masks. We generated the tRNA ${ }^{\text {Asp }}$ model using S2 $\mathrm{S}^{34}$, based on the similarity of tRNA ${ }^{\text {Asp }}$ with $\mathrm{tRNA}^{\mathrm{fMet}}$ (and P-site codon) from the $\mathrm{X}$-ray structure of the Thermus thermophilus programmed $70 \mathrm{~S}$ ribosome ${ }^{35}$. We constructed structure-based sequence alignments using S2 $\mathrm{S}^{34}$ based on similarity of $28 \mathrm{~S}$ rRNA with the $23 \mathrm{~S}$ rRNA of Haloarcula marismortui ${ }^{2}$ and E. coli $50 \mathrm{~S}$ subunits ${ }^{36}$. We generated the models using an extended version of MANIP ${ }^{37}$. We refined and fitted the generated models using RNAVIEW ${ }^{38}$ and $\mathrm{MDFF}^{39}$. We built protein alignments with TCoffee ${ }^{40}$. We built Homology models for ribosomal proteins using Modeler ${ }^{41}$. We then adjusted the models manually with $\operatorname{Coot}^{42}$ and minimized them with $\mathrm{VMD}^{43}$. We modeled the CCA-Asp115 position of the helix nascent chains based on an alignment with the H. marismortui 50 S subunit in complex with CCA-pcb ${ }^{24,44}$, where we obtained the Asp115 by simple mutation of phenylalanine in the CCA-pcb ligand. We performed initial docking of $\mathrm{X}$-ray structures and models using Chimera ${ }^{45}$ and alignment of PDB structures was performed using PyMol (http://www.pymol.org). All figures were generated using Chimera ${ }^{45}$ and PyMol.

30. Erickson, A.H. \& Blobel, G. Cell-free translation of messenger RNA in a wheat germ system. Methods Enzymol. 96, 38-50 (1983).

31. Wagenknecht, T., Frank, J., Boublik, M., Nurse, K. \& Ofengand, J. Direct localization of the tRNA-anticodon interaction site on the Escherichia coli $30 \mathrm{~S}$ ribosomal subunit by electron microscopy and computerized image averaging. J. Mol. Biol. 203, 753-760 (1988).

32. Mindell, J.A. \& Grigorieff, N. Accurate determination of local defocus and specimen tilt in electron microscopy. J. Struct. Biol. 142, 334-347 (2003).

33. Frank, J. Three-dimensional electron microscopy of macromolecular assemblies. in Three-dimensional Electron Microscopy of Macromolecular Assemblies (Academic Press, 1996).

34. Jossinet, F. \& Westhof, E. Sequence to structure (S2S): display, manipulate and interconnect RNA data from sequence to structure. Bioinformatics 21, 3320-3321 (2005).

35. Selmer, M. et al. Structure of the 70S ribosome complexed with mRNA and tRNA. Science 313, 1935-1942 (2006).

36. Schuwirth, B.S. et al. Structures of the bacterial ribosome at $3.5 \AA$ resolution. Science 310, 827-834 (2005).

37. Massire, C. \& Westhof, E. MANIP: an interactive tool for modelling RNA. J. Mol. Graph. Model. 16, 197-205, 255-197 (1998).

38. Yang, H. et al. Tools for the automatic identification and classification of RNA base pairs. Nucleic Acids Res. 31, 3450-3460 (2003).

39. Trabuco, L.G., Villa, E., Mitra, K., Frank, J. \& Schulten, K. Flexible fitting of atomic structures into electron microscopy maps using molecular dynamics. Structure 16, 673-683 (2008).

40. Notredame, C., Higgins, D.G. \& Heringa, J. T-Coffee: a novel method for fast and accurate multiple sequence alignment. J. Mol. Biol. 302, 205-217 (2000).

41. Eswar, N. et al. Comparative protein structure modeling using Modeller. Curr. Protoc. Bioinformatics 5, 5.6 (2006).

42. Emsley, P. \& Cowtan, K. Coot: model-building tools for molecular graphics. Acta Crystallogr. D Biol. Crystallogr. 60, 2126-2132 (2004).

43. Humphrey, W., Dalke, A. \& Schulten, K. VMD: visual molecular dynamics. J. Mol. Graph. 14, 33-38 (1996).

44. Schmeing, T.M., Huang, K.S., Kitchen, D.E., Strobel, S.A. \& Steitz, T.A. Structural insights into the roles of water and the $2^{\prime}$ hydroxyl of the P site tRNA in the peptidyl transferase reaction. Mol. Cell 20, 437-448 (2005).

45. Pettersen, E.F. et al. UCSF Chimera: a visualization system for exploratory research and analysis. J. Comput. Chem. 25, 1605-1612 (2004). 